\title{
Optimization of preparation of chitosan-coated iron oxide nanoparticles for biomedical applications by chemometrics approaches
}

\author{
Soheila Honary ${ }^{1}$, Pouneh Ebrahimi ${ }^{*}$, Hossein Asgari Rad ${ }^{1}$ and Mahsa Asgari ${ }^{1}$
}

\begin{abstract}
Functionalized magnetic nanoparticles are used in several biomedical applications, such as drug delivery, magnetic cell separation, and magnetic resonance imaging. Size and surface properties of iron oxide nanoparticles are the two important factors which could dramatically affect the nanoparticle efficiency as well as their stability. In this study, the chemometrics approach was applied to optimize the coating process of iron oxide nanoparticles. To optimize the size of nanoparticles, the effect of two experimental parameters on size was investigated by means of multivariate analysis. The factors considered were chitosan molecular weight and chitosan-to-tripolyphosphate concentration ratio. The experiments were performed according to face-centered cube central composite response surface design. A second-order regression model was obtained which characterized by both descriptive and predictive abilities. The method was optimized with respect to the percent of $Z$ average diameter's increasing after coating as response. It can be concluded that experimental design provides a suitable means of optimizing and testing the robustness of iron oxide nanoparticle coating method.
\end{abstract}

Keywords: Experimental design; Optimization; Nanoparticles; Iron oxide; Coating

\section{Background}

Magnetic nanoparticles (MNPs) are a major class of nanoscale materials with the potential to revolutionize current clinical diagnostic and therapeutic techniques. MNPs are being actively investigated as the next generation of magnetic resonance imaging contrast agents and as carriers for targeted drug delivery due to their unique physical properties and ability to function at the cellular and molecular level of biological interactions [1-3]. Composition, size, morphology, and surface chemistry can now be tailored by various processes to not only improve magnetic properties but also affect the behavior of nanoparticles in vivo $[4,5]$. In its simplest form, a biomedical MNP platform is comprised of an inorganic nanoparticle core and a biocompatible surface coating that provides stabilization under physiological conditions $[6,7]$. With the ability to utilize magnetic attraction and/

\footnotetext{
* Correspondence: epouneh@yahoo.com

2Department of Chemistry, Faculy of Basic Sciences, Golestan University,

P. O. Box 155, Gorgan 4913815759, Iran

Full list of author information is available at the end of the article
}

or specific targeting of disease biomarkers, MNPs offer an attractive means of remotely directing therapeutic agents specifically to a disease site while simultaneously reducing dosage and the deleterious side effects associated with non-specific uptake of cytotoxic drugs by healthy tissue. These physicochemical properties of nanoparticles directly affect their subsequent pharmacokinetics and biodistribution [8]. To increase the effectiveness of MNPs, several techniques, including reducing size and grafting non-fouling polymers, have been employed to improve their stealthiness and increase their blood circulation time to maximize the likelihood of reaching targeted tissues $[9,10]$. Polymeric coating provides a steric barrier to prevent nanoparticle agglomeration and avoid opsonization. In addition, these coatings provide a means to tailor the surface properties of MNPs such as surface charge and chemical functionality. Some critical aspects with regard to polymeric coatings that may affect the performance of an MNP system include the nature of the chemical structure of the polymer (e.g., hydrophilicity/hydrophobicity, biodegradation characteristics, etc.), the length or molecular

\section{实


Table 1 Two studied factors and the corresponding three level settings

\begin{tabular}{ccc}
\hline Level & MW & Chi/TPP (ratio) \\
\hline-1 & LMW $^{\mathrm{a}}$ & $2: 1$ \\
0 & MMW $^{\mathrm{b}}$ & $4: 1$ \\
+1 & $\mathrm{HMW}^{\mathrm{c}}$ & $6: 1$ \\
\hline${ }^{\text {a Low molecular weight; }}{ }^{\mathrm{b}}$ medium molecular weight; ${ }^{\text {chigh molecular weight. }}$
\end{tabular}

weight of the polymer, the manner in which the polymer is anchored or attached (e.g., electrostatic or covalent bonding), the conformation of the polymer, and the degree of particle surface coverage [11]. For drug delivery applications, iron oxide MNP must be precoated with substances that assure their stability, biodegradability, and non-toxicity in the physiological medium in order to achieve combined properties of high magnetic saturation, biocompatibility, and interactive functions on the surface [12]. The surfaces of these particles could be modified through a coating process by depositing a few atomic layers of biocompatible polymers. The polymer coating not only leads to the creation of more hydrophilic nanostructures but also provides a variety of surface functional groups to bind drug molecules, inhibit aggregation, and increase stability [5]. Both natural and synthetic polymers have been used in the preparation of coated MNP [3]. Chitosan is a polysacharide produced by deacetylation of chitin naturally extracted from shells of crabs and shrimps. Chitosan is a unique cationic, hydrophilic polymer that beneficial properties, such as low toxicity, low immunogenicity, excellent biodegradability, and biocompatibility as well as a high positive charge that easily forms polyelectrolyts complexes with negatively charged polyanions. It is well known that the amine group on chitosan molecules may interact with

Table 2 Experimental conditions for face-centered cube central composite design and average response for particle size

\begin{tabular}{cccc}
\hline Trial & MW & Chi/TPP (ratio) & Increased size (\%) \\
\hline 1 & -1 & -1 & 96.0 \\
2 & -1 & +1 & 76.0 \\
3 & +1 & -1 & 91.0 \\
4 & +1 & +1 & 223.8 \\
5 & -1 & 0 & 116.0 \\
6 & +1 & 0 & 138.0 \\
7 & 0 & -1 & 78.0 \\
8 & 0 & +1 & 118.0 \\
9 & 0 & 0 & 97.0 \\
10 & 0 & 0 & 87.0 \\
11 & 0 & 0 & 65.0 \\
\hline
\end{tabular}

Table 3 Intercept, coefficients, mean effects, and statistics of the predictive model obtained for the increased size of iron oxide nanoparticles

\begin{tabular}{lcc}
\hline Variable & Coefficient & Mean effect $(\boldsymbol{p} \text { value })^{\mathbf{a}}$ \\
\hline Intercept & 89.000 & - \\
$\mathrm{MW}$ & 27.467 & $0.485(0.008)$ \\
$(\mathrm{Chi} / \mathrm{TPP})$ & 25.467 & $0.450(0.011)$ \\
$\mathrm{MW}^{2}$ & 34.467 & $0.410(0.016)$ \\
$\mathrm{MW} \times($ Chi/TPP $)$ & 38.200 & $0.551(0.004)$ \\
$R$ & & 0.954 \\
$\mathrm{SE}^{\mathrm{b}}$ & & 17.060 \\
$F^{\mathrm{c}}(p$ value $)$ & & $15.025(0.003)$ \\
\hline
\end{tabular}

${ }^{a} p$ value is $0.05 ;{ }^{\mathrm{b}}$ standard error of the estimate; ${ }^{\mathrm{C}}$ Fisher ratio.

$\mathrm{Fe}_{3} \mathrm{O}_{4}$ [13]. Optimization in the field of formulation especially formulation of nanosystems is often done according to traditional methods which are based on changing one variable at a time [14]. To optimize a formulation process, various experimental design methods can be used $[15,16]$. Optimization of nanoparticle preparation can be difficult due to the wide array of parameters and variables which must be controlled to achieve specific particle size. A face-centered cube central composite response surface design can be used to evaluate the relationship between one or more response variables and a set of quantitative experimental parameters [17] (Additional file 1).

The objectives of this work are to optimize the coating process of $\mathrm{Fe}_{3} \mathrm{O}_{4}$ nanoparticles (Fe NP) with chitosan and to create a model revealing the influence of various factors on the size of iron oxide nanoparticles after coating.

\section{Methods}

$\mathrm{FeCl}_{3} \cdot 6 \mathrm{H}_{2} \mathrm{O}, \mathrm{FeCl}_{2} \cdot 4 \mathrm{H}_{2} \mathrm{O}, \mathrm{NaCl}$, and $\mathrm{NaOH}$ were obtained from Merck (Darmstadt, Germany). Low, medium, and high molecular weights of chitosan with MWs of 100, 400, and $600 \mathrm{kD}$, respectively, were purchased from Fluka BioChemica (Tokyo, Japan). The degree of deacetylation was $85 \%$.

\section{Preparation of nanoparticles}

Iron oxide nanoparticles were prepared by adding a base to an aqueous mixture of $\mathrm{Fe}^{2+}$ and $\mathrm{Fe}^{3+}$ chloride at 0.5 molar ratio. In this method, the $\mathrm{pH}$ value of the $\mathrm{Fe}^{2+}$ and $\mathrm{Fe}^{3+}$ solution (concentration ratio 1:2) was adjusted at 10 by $2 \mathrm{~N} \mathrm{NaOH}$ under $\mathrm{N}_{2}$ gas. The solution was continuously agitated using a magnetic stirrer to produce a black precipitate. Then, precipitate was collected and washed by deionized water. Equation 1 shows the related reaction:

$$
\mathrm{Fe}^{2+}+2 \mathrm{Fe}^{3+}+8 \mathrm{OH}^{-} \rightarrow \mathrm{Fe}_{3} \mathrm{O}_{4}+4 \mathrm{H}_{2} \mathrm{O}
$$




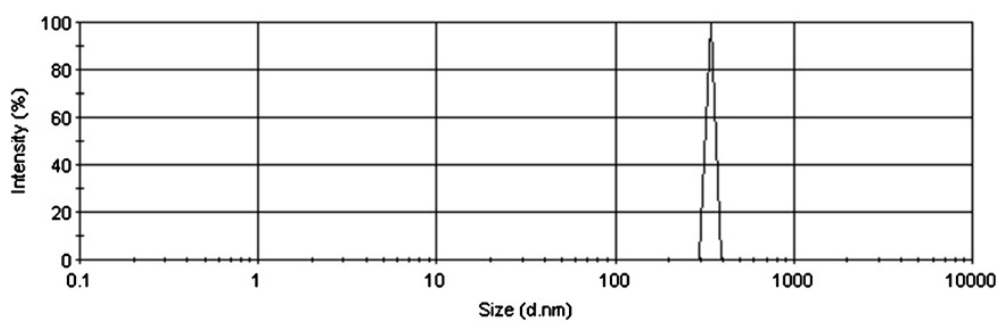

Figure 1 Particle size distribution for low molecular weight condition of chitosan (100 kD) and Chi/TPP ratio (5.5:1).

According to the thermodynamic of the reaction, a complete precipitation of $\mathrm{Fe}_{3} \mathrm{O}_{4}$ should be expected under a non-oxidizing (oxygen-free) environment. Otherwise, $\mathrm{Fe}_{3} \mathrm{O}_{4}$ might also be oxidized to $3 \mathrm{Fe}(\mathrm{OH})_{3}$. Thus, the synthesis was done in an oxygen-free environment by passing $\mathrm{N}_{2}$ gas. Chitosan solutions (Chi) with different concentrations and molecular weights were prepared by dissolving chitosan in 1\% (W/V) acetic acid solution using sonication until the solution was transparent. Tripolyphosphate (TPP) was desolved in deionized water. The iron nanoparticles were dispersed in chitosan solution. TPP solution was also added to the mixture. The percent of $\mathrm{Z}$ average diameter (ZAD) increasing of Fe nanoparticles after coating them with chitosan was calculated by following equation:

$$
\begin{gathered}
\text { ZAD increasing } \%=(\text { ZAD of FeNP after coating-ZAD } \\
\text { of FeNP befor coating }) \\
\times 100 / \text { ZAD of FeNP befor coating }
\end{gathered}
$$

\section{Evaluation of nanoparticles}

Size and polydispersity of particles were determined by a Zetasizer Nano Particle Analyzer using Zetasizer 3600 at $25^{\circ} \mathrm{C}$ with a scattering angle of $90^{\circ}$ (Malvern Instruments, Malvern, UK). The analysis of the surface and

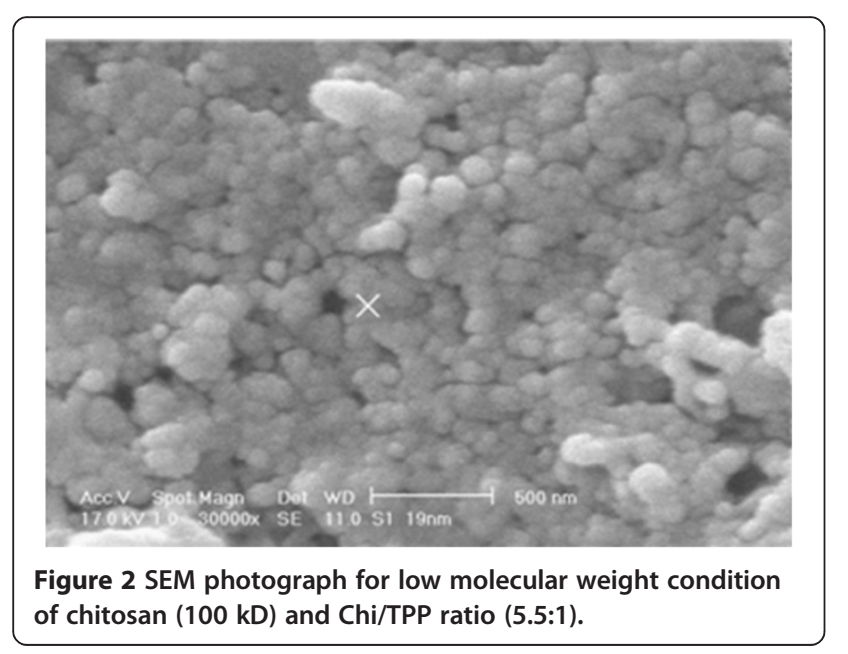

shape characteristics of the nanoparticles were determined using a scanning electron microscope (model 2360, SEM; Leo Oxford, England) after coating.

\section{Experimental design}

In this study, two experimental parameters were varied at three levels according to Table 1 . These parameters were chosen as they were considered to have the most significant effect on the size of nanoparticles. The levels were selected based on knowledge that acquired from initial experimental trials. All experiments were carried out in duplicate. A two factor, at three levels, facecentered cube central composite response surface design was also used for testing the robustness of the method. The experiment in the central point provided a more precise estimate of pure experimental error and provided a measure for the adequacy of the model (lack of fit). All statistical analysis was performed on range scaled factor values of $[-1,+1]$ with SPSS software (IBM Corp., Armonk, NY, USA).

\section{Results and discussion}

The effect of two factors on $\mathrm{Z}$ average diameter of nanoparticles was studied. The factors considered were chitosan-to-TPP concentration ratio (Chi/TPP ratio) and chitosan molecular weight. The experiments were performed in a random order on three levels for each factor using a face-centered cube central composite response surface design (Table 2) that provides enough information for calculation of the regression model containing linear, interactions, and curved factor effects. The values of response data obtained for the experiments were also presented in Table 2.

The increasing of $\mathrm{Z}$ average diameter of nanoparticles (\%) after coating for all 11 experiments was fitted to the polynomial model. The coefficients of the model were calculated by backward multiple linear regression technique and validated by the analysis of variance (ANOVA). The criteria for the evaluation of descriptive capability of a polynomial were Fisher ratio value $(F)$, correlation coefficient $(R)$, and standard error of the estimate (SE). It was found that the model which successfully described the size increasing (\%) of nanoparticles 


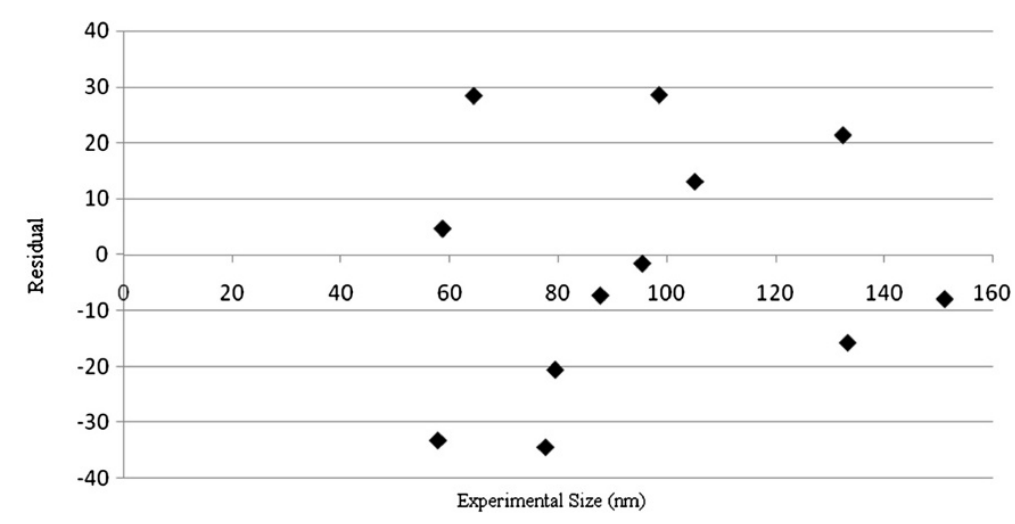

Figure 3 Residual plot for 13 experimental conditions not entered in the modeling.

was a simple polynomial containing the main effect of two factors, interaction, and the square term of chitosan molecular weight. The related model and its statistics are presented in Table 3.

The results of ANOVA demonstrated that the largest effect on size increasing during coating process of nanoparticles was due to the interaction term between the main factors with $p$ value 0.004 and mean effect 0.551 . The mean effect $p(<0.05)$ values show that this term is completely significant in the MLR model. Also, the significant effect of two factors MW and Chi/TPP ratio in the model (with the $p$ value 0.008 and 0.011 , respectively) shows that these factors influence the $\mathrm{Z}$ average size of nanoparticles and describe response well. It is interesting to demonstrate that in this study, the quadratic term of MW was also significant in the size increasing of nanoparticles ( $p$ value 0.016 ) while no significant effect was observed due to the square term of Chi/TPP. This means that the existence of interaction between the principal factors in conditions of our experiments and $\mathrm{MW}^{2}$ emphasizes once the necessity to carry out active multi-factor experiments for optimization of the preparation process of nanoparticles. Therefore, the use of simultaneous method is essential for optimization of iron oxide nanoparticle coating process.

Then, the size of nanoparticles was predicted in other experimental conditions using this simple equation. Then, the best obtained conditions were tested and the experimental results compared to predicted results. The graph of particle size distribution under one of the predicted condition (low MW and Chi/TPP ratio, 5.5:1) and SEM photograph were shown in Figures 1 and 2, respectively.

Under this condition, the predicted and experimental increased size (\%) was $77.7 \%$ and $112 \%$, and the calculated particle size also were 446.1 and $532.3 \mathrm{~nm}$, respectively. Also, to assess the suitability of obtained MLR model, the residuals were analyzed statistically. Figure 3

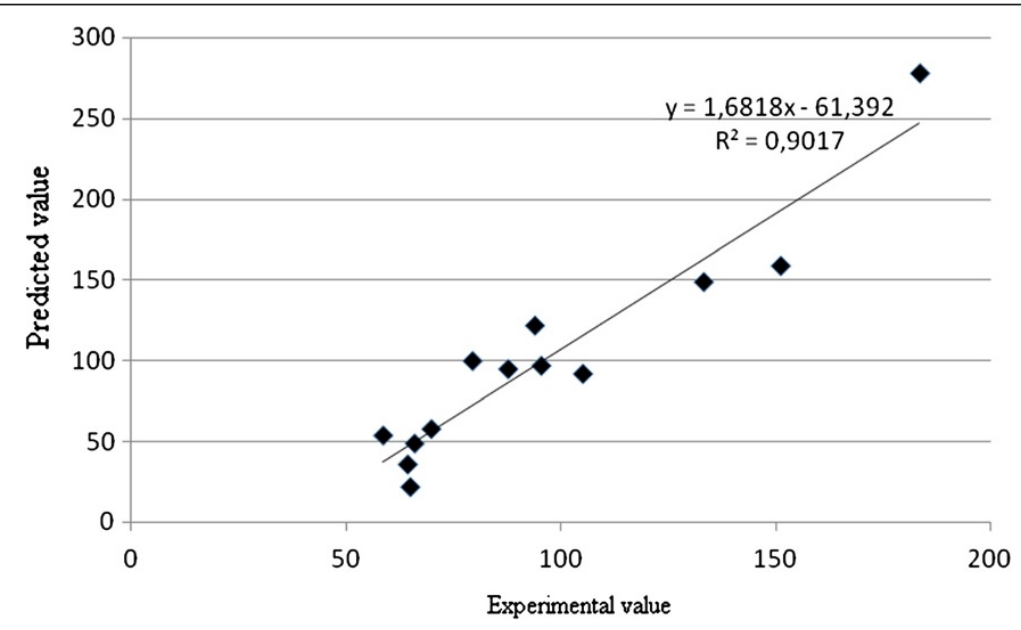

Figure 4 Predicted vs. experimental iron oxide nanoparticle size increasing (\%) for 13 experimental conditions not entered in the modeling. According to the regression model reported in Table 3 (these conditions not entered in the modeling). 
also shows a good agreement between predicted and experimental results for 13 experimental conditions not entered in the modeling. From the plot of Figures 3 and 4 , it can be observed that the fitting of this model and the model prediction ability is quite satisfactory.

\section{Conclusions}

Face-centered cube response surface experimental design in conjunction with soft modeling has been shown to be efficient in mapping response surface for changing the percent of particle size increasing. The results showed that a simple MLR model can be successfully used to evaluate the increasing of particle size and to search an optimum set of experimental conditions. To find the optimal conditions of particle size increasing (\%), a second-order polynomial equation was generated to modeling the particle size increasing as a function of Chi/TPP ratio and chitosan molecular weight. The efficiency of the prediction of the model was confirmed by performing the experiment under the optimal condition.

\section{Additional file}

Additional file 1: Chemometric approach was applied to optimize the coating process of iron oxide nanoparticles. To optimize, the effect of two experimental parameters, chitosan molecular weight (MW) and chitosan concentration, was investigated on size by mean of multivariant analysis.

\section{Competing interests}

The authors declare that they have no competing interests.

\section{Authors' contributions}

All authors read and approved the final manuscript.

\section{Author details}

'Department of Pharmacy, School of Pharmacy, Mazandaran University of Medical Sciences, Sari, 48189, Iran. ²Department of Chemistry, Faculy of Basic Sciences, Golestan University, P. O. Box 155, Gorgan 4913815759, Iran.

\section{Received: 3 February 2013 Accepted: 10 July 2013}

Published: 23 Aug 2013

\section{References}

1. Corrot, C, Robert, P, Idee, JM, Port, M: Recent advances in iron oxide nanocrystal technology for medical imaging. Adv. Drug Deliver. Rev. 58, 14711504 (2006)

2. Pankhurst, QA, Connolly, J, Jones, SK, Dobson, J: Applications of magnetic nanoparticles in biomedicine. J. Phys. D: Appl. Phys. 36, R167-181 (2003)

3. Dobson, J: Magnetic nanoparticles for drug delivery. Drug Develop Res 67, 55-60 (2006)

4. Tartaj, P, Morales, MD, Veintemillas-Verdaguer, S, Gonzalez-Carreno, T, Serna, $\mathrm{CJ}$ : The preparation of magnetic nanoparticles for applications in biomedicine. J. Phys. D: Appl. Phys. 36, R182-197 (2003)

5. Gupta, AK, Gupta, M: Synthesis and surface engineering of iron oxide nanoparticles for biomedical applications. Biomaterials 26, 3995-4021 (2005)

6. McNeil, SE, Leukocyte, J: Nanotechnology for the biologist. Biol. 78, 585-594 (2005)

7. Frullano, L, Meade, TJ: Multimodal MRI contrast agents. J. Biol. Inorg. Chem. 12, 939-949 (2007)

8. Chouly, C, Pouliquen, D, Lucet, I, Jeune, J, Jallet, P: Development of superparamagnetic nanoparticles for MRl: effect of particle size, charge and surface nature on biodistribution. J. Microencapsul. 13, 245-255 (1996)
9. Gref, R, Luck, M, Quellec, P, Marchand, M, Dellacherie, E, Harnisch, S, Blunk, T, Muller, RH: Muller, 'Stealth' corona-core nanoparticles surface modified by polyethylene glycol (PEG): influences of the corona (PEG chain length and surface density) and of the core composition on phagocytic uptake and plasma protein adsorption, Colloids Surf. Colloid Surface B. 18, 301-313 (2000)

10. Moghimi, SM, Hunter, AC, Murray, JC: Long-circulating and target-specific nanoparticles: theory to practice. Pharmacol. Rev. 53, 283-318 (2001)

11. Berry, CC, Curtis, ASG: Functionalisation of magnetic nanoparticles for applications in biomedicine. J. Phys. D: Appl. Phys. 36, R198-206 (2003)

12. Tartaj, P, Morales, M, Gonzalez-Carreno, T, Veintemillas-Verdaguer, S, Serna, C: Advances in magnetic nanoparticles for biotechnology applications. J. Magn. Magn. Mater. 290, 28-34 (2005)

13. Majeti, NV, Kumar, R: A review of chitin and chitosan applications. React. Funct. Polym. 46, 1-27 (2000)

14. Bouchemal, K, Briancon, S, Perrier, E, Fessi, H: Nano-emulsion formulation using spontaneous emulsification: solvent, oil and surfactant optimisation. Int. J. Pharm. 280, 241-251 (2004)

15. Chopra, S, Patil, GV, Motwani, SK: Motwani, Release modulating hydrophilic matrix systems of losartan potassium: Optimization of formulation using statistical experimental design Eur. J. Pharm. 66, 73-82 (2007)

16. Ki Do, K, Han, DN, Kim, HT: Optimization of experimental conditions based on the Taguchi robust design for the formation of nano-sized silver particles by chemical reduction method. Chem. Eng. J. 104, 55-61 (2004)

17. Gonzalez, AG: Optimization of pharmaceutical formulations based on response-surface experimental design. Int. J. Pharm. 97, 149-159 (1993)

10.1186/2228-5326-3-48

Cite this article as: Honary et al:: Optimization of preparation of chitosan-coated iron oxide nanoparticles for biomedical applications by chemometrics approaches. International Nano Letters 2013, 3:48

\section{Submit your manuscript to a SpringerOpen ${ }^{\odot}$ journal and benefit from:}

- Convenient online submission

Rigorous peer review

- Immediate publication on acceptance

- Open access: articles freely available online

- High visibility within the field

- Retaining the copyright to your article

Submit your next manuscript at springeropen.com 